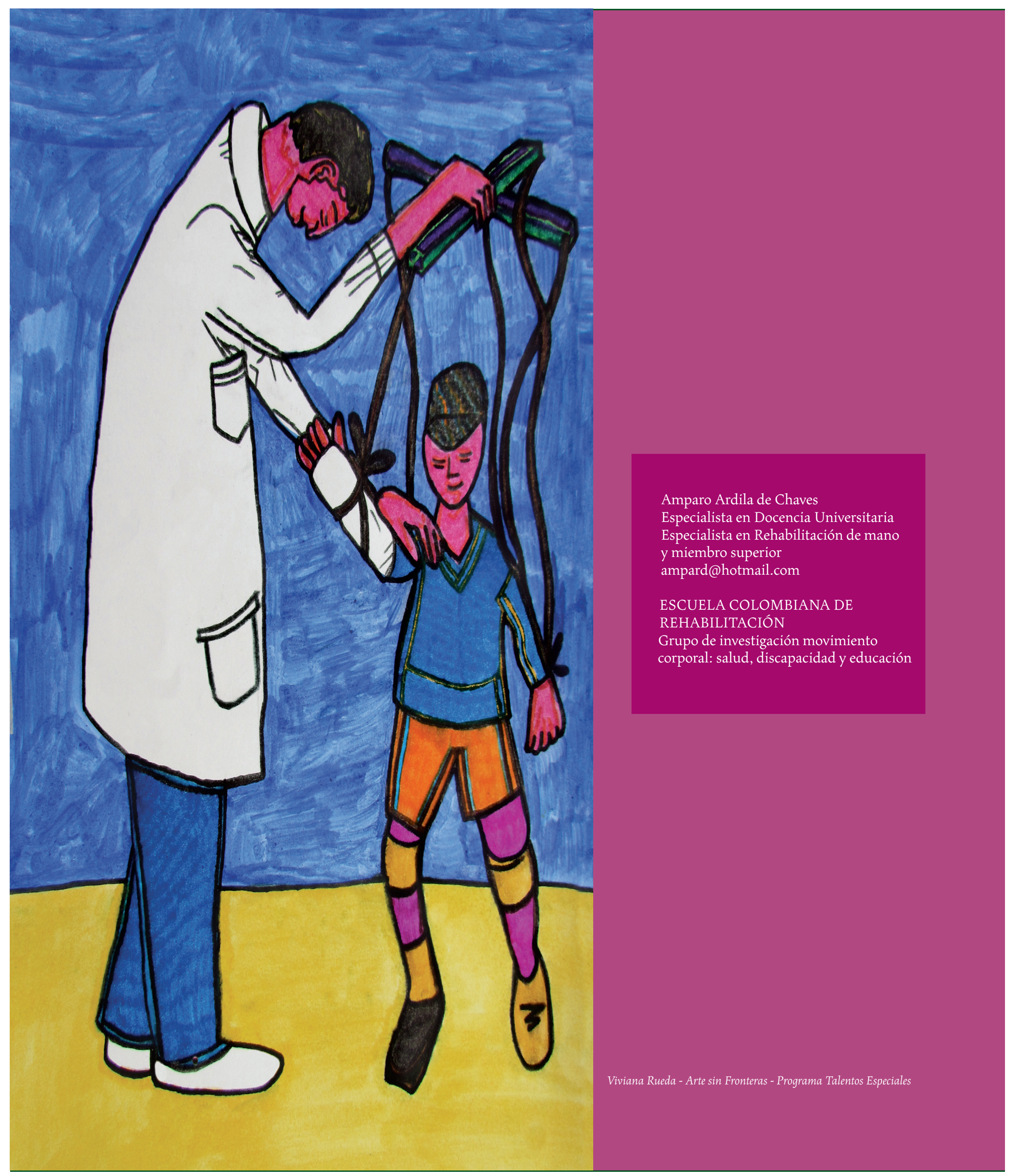




\title{
PERTINENCIA Y OPORTUNIDAD DE TERAPÉUTICA ESPECIALIZADA A PACIENTES CON COMPROMISO FUNCIONAL DE MIEMBRO SUPERIOR
}

\section{Relevance and Opportunity of Therapeutic Specialized for Patients With Functional Limitations of Upper Limb}

Fecha de Recepción: 5 de Junio de 2011 - Fecha de Aprobación: 15 de Agosto de 2011

\begin{abstract}
RESUMEN
La creación de la Ley 100 (1), tuvo dentro de sus objetivos lograr la calidad y la equidad en la atención en salud de los colombianos, sin restricciones económicas ni sociales para acceder a todos los servicios requeridos. El compromiso de función de un segmento como el miembro superior requiere de atención especializada, de forma pertinente y oportuna, por ser un organismo complejo interrelacionado en todos sus componentes aunque aparentemente no haya conexión entre unos y otros (2). En una lesión, la meta terapéutica debe encaminarse al reintegro del paciente a su rol personal, laboral y de su entorno. El objetivo de este estudio fue determinar la oportunidad y la pertinencia de la terapéutica especializada prestada por los diferentes regímenes de atención en salud, a pacientes colombianos con compromiso de función en miembro superior. Se hizo una investigación exploratorio-descriptiva llevada a cabo en diez ciudades colombianas2, en donde se aplicó un formato previamente piloteado, a 135 pacientes del régimen contributivo, 60 de régimen subsidiado y a 82 de medicina prepagada. Se estableció que el 100\% de los pacientes con patología de miembro superior que comprometía su función atendidos en los tres regímenes de salud, no recibieron atención terapéutica especializada, aunque si fueron remitidos de forma oportuna a terapia por el médico que los atendió inicialmente, pues el 52\% de los pacientes del régimen subsidiado, el $68 \%$ del contributivo y el $94 \%$ de la prepagada recibieron atención entre el $1^{\circ}$ y el $5^{\circ}$ día posterior a la consulta.
\end{abstract}

PALABRAS CLAVE

Pertinencia, oportunidad, terapéutica especializada, compromiso funcional de miembro superior.

\section{ABSTRACT}

The creation of Act 100 (1), had within its objectives to achieve quality and equity in the health care of Colombians, unrestricted economic or social to access all required services. The limitation of a segment as the upper limb function requires specialized care, relevant and timely, as a complex organism interrelated in all its components although apparently there is no connection between the two (2). In an injury, therapeutic goal should aimed at the reinstatement of the patient to his role as personal, employment and environment. The objective of this study was to determine the opportunity and relevance of the specialized Therapeutics provided by different health care regimens, Colombian patients with upper limb functional limitation. Became a descriptive-exploratory research carried out in ten Colombian cities 2, applied a format previously piloted 135 patients of the contributory scheme, 60 of subsidized regime and 82 of prepaid medicine. It was established that $100 \%$ of patients with pathology of upper limb that compromised their role served in the three regimes of health, did not receive therapeutic care specialized, although if they were submitted in a timely manner to therapy by the physician that attended them initially, since $52 \%$ of patients of the subsidized regime, $68 \%$ of the contributory and $94 \%$ of the prepaid receive attention between the 1st and the 5 th day after the consultation.

KEY WORDS

Relevance, opportunity, specialized Therapeutics, upper limb functional commitment. 


\section{INTRODUCCIÓN}

L a salud es una de las condiciones más importantes de la vida humana y un componente fundamental de las oportunidades humanas que se deben valorar, para alcanzar una vida sana y sobre la cual existen muchas influencias, como el entorno epidemiológico y las especificidades laborales $(3,4)$.

En América Latina según informe del Programa de las Naciones Unidas para el Desarrollo (5) (PNUD), los esfuerzos por mejorar la calidad de vida de los colectivos, han tenido repercusiones importantes a nivel del mundo, aunque se dimensiona un gran rezago respecto de los países industrializados, lo cual lleva a profundas inequidades, y es por ello que se hace necesario que las comunidades que trabajan en salud investiguen para conocer las debilidades existentes en atención y aporten al mejoramiento de la salud de su entorno social $(3,6)$

La búsqueda de la equidad en salud en los países no industrializados ha sido una constante, aunque autores como Moran (7), han establecido que los sistemas de salud presentan siempre inequidades y que un mayor número de servicios y de mejor calidad gravitan en torno a las personas acomodadas, que los necesitan menos que la población pobre y marginada.

Amartya Sen (3) dice que la salud y la enfermedad deben tener un lugar destacado en discusiones sobre equidad y justicia social. En Colombia con la Ley 100 (1) se quiso garantizar el cubrimiento en salud de forma justa e igualitaria en donde se incluyera a los más pobres y vulnerables y así encontrar una salida a la problemática social existente, para lo cual se constituyeron los regímenes contributivo y subsidiado y a la par se creó una estructura de prestación de servicios prepagados.

El subsidiado, definido en el artículo 211 de la misma ley 100 , como "un conjunto de normas que rigen la vinculación de los individuos al sistema general de seguridad social en salud, cuando tal vinculación se hace a través del pago de una cotización subsidiada, total o parcialmente, con recursos fiscales o de solidaridad". Está fundamentado en el principio de la solidaridad en donde los que tienen mejor condición económica ayudan a los más pobres. Este Sistema de Selección de Beneficiarios (SISBEN) establece niveles del 1 al 3 según el recurso económico con que cuente cada persona.

De acuerdo con la Ley 1122 de 2007 (8) éstos afiliados tienen derecho a "todas las acciones de rehabilitación", lo cual incluye la intervención terapéutica especializada y además de "recibir atención con calidad y así evitar complicaciones o consecuencias graves".

En el artículo 202 de la misma Ley 100 se define el régimen contributivo como "el conjunto de normas que rigen la vinculación de los individuos y las familias al Sistema General de Seguridad Social en Salud (SGSSS), cuando tal vinculación se hace a través del pago de una cotización individual y familiar, o un aporte económico previo, financiado directamente por el afiliado o en congruencia entre éste y su empleador".

Para cubrimiento de éste régimen se creó el Plan Obligatorio de Salud (POS) que proporciona servicios básicos a los usuarios afilia- dos, en las áreas de recuperación de la salud, prevención de la enfermedad y cubrimiento de ingresos de manera transitoria, como prestaciones económicas, cuando ocurren incapacidades por enfermedad, accidentes o maternidad.

El inicio de la Medicina Prepagada se dio para satisfacer las necesidades de las comunidades que por deficiencias en los servicios de sus regímenes de salud, sentían limitada su cobertura. Las entidades que la conformaron, inicialmente desarrollaron su actividad sin limitación de normas administrativas o de vigilancia y sólo se dieron los primeros pasos con la expedición de los Decretos 800 de 1992, 1570 de 1993 y 1486 de 1994.

Cada entidad de salud que ofrece los servicios de medicina prepagada, establece las condiciones de alcances y características en prestación de su servicio y dispone para libre elección de los usuarios, centros de diagnóstico y grupos de profesionales que cubren áreas específicas y a los que se puede acceder, en la mayoría de las entidades de forma directa, es decir sin remisión de otro profesional. Las tarifas de los bonos que son similares a los copagos de las EPS, las establecen las entidades de medicina prepagada, con vigilancia del Ministerio de Salud

Actualmente el Ministerio de Salud es el ente regulador del Sistema de Protección Social, del SGSSS y el que tiene la función de proveer de manera integral, las acciones de salud, individuales y colectivas con la participación responsable de todos los sectores de la sociedad.

La normativa que rige la salud de los colombianos ha establecido que la calidad y la equidad en la atención de los individuos son requerimientos obligatorios para proporcionarles calidad de vida, y uno de los parámetros de medición de éstos, es la oportunidad que tienen las personas para acceder a los diferentes servicios de salud en un tiempo justo para evitar complicaciones, y que incluya los especializados, (pertinencia) con profesionales idóneos sin que existan restricciones, en ninguno de los regímenes establecidos en la legislación colombiana, proporcionándole al paciente la intervención adecuada en tiempo, frecuencia y cantidad para que recupere su integralidad (1).

La Ley también establece que la oportunidad para acceder a los servicios de salud y de asistencia médica no deben estar sometidos a retrasos innecesarios o injustificados y que sobre los aseguradores y prestadores de salud, recae la carga de la atención diligente (1).

El Decreto 1011 de 2006, del Ministerio de Protección social de Colombia, estableció el Sistema Obligatorio de Garantía de Calidad en Salud, que rige a las Empresas Administradoras de Planes de Beneficios (EAPB), como son las Entidades Promotoras de Salud de los Regímenes Subsidiado (Administradoras del Régimen Subsidiado), y Contributivo, Entidades Adaptadas y Empresas de Medicina Prepagada (9) y aunque la afiliación ha ido en aumento, hay diferencias significativas en aspectos sociodemográficos y geográficos, como lo refiere Andrés Zambrano (10), pues en el 2003 en el área rural, el $35 \%$ estaba asegurado al régimen subsidiado y el $12 \%$ al régimen contributivo, lo cual confirma que en la actualidad estas diferencias persisten. 
La pertinencia y la oportunidad en la atención a los usuarios por diferentes patologías, por parte de los regímenes subsidiados, contributivos o prepagados, está por determinarse pues estudios como el realizado por Julián Vargas (11) establecen que "el aseguramiento se convirtió en un fin en sí mismo y estar afiliado al SGSSS no garantiza el acceso efectivo a los servicios", y por consiguiente a la calidad en la atención, prestada por éstos, dentro de la cual está contemplada la pertinencia y la oportunidad en la atención a los usuarios y en este contexto a la terapéutica especializada proporcionada a los usuarios del sistema de salud colombiano.

Hay escritos que establecen que aunque no existe una definición que determine con claridad cuáles son los tiempos oportunos, en países como Italia(12) se definen plazos de oportunidad casi iguales a los establecidos en Colombia por la reglamentación interna de empresas aseguradoras de carácter privado como Compensar y Solsalud, y gubernamentales como la Caja de Previsión Social (Caprecom), en donde se estima que no es oportuno el otorgamiento de citas con el especialista cuando se da posterior a 8 días de su solicitud(13) y Caja Nacional de Previsión Social (Cajanal) en donde según resolución 000707(14) se establece un tiempo máximo de 5 días hábiles siguientes a la solicitud, para recibir atención especializada.

En este trabajo se tomó la definición de oportunidad como la posibilidad que tiene el usuario de obtener los servicios que requiere sin que se presenten retrasos que pongan en riesgo su vida o su salud. Esta característica se relaciona con la organización de la oferta de servicios en relación con la demanda y con el nivel de coordinación institucional para gestionar el acceso a los servicios. (9)

Para evaluar esta variable se agruparon las preguntas referentes al tiempo transcurrido entre la solicitud de consulta con el terapeuta y la asignación de la cita, la cantidad de sesiones de terapia autorizadas y el número de sesiones de tratamiento a las que asistió. Se establecieron rangos en número de días, de 1 a 5, (Muy oportuno), de 6 a 10 (Oportuno), de 11 a 15 (Poco oportuno) y de 15 a 20 (No oportuno) (13).

En cuanto a pertinencia se definió como el grado en el cual los usuarios obtienen los servicios que requieren, con la mejor utilización de los recursos (humanos, en este contexto) de acuerdo con la evidencia científica, para que los efectos secundarios sean menores que los beneficios potenciales. (9)

Esta variable se operacionalizó con las preguntas de que si posterior al tratamiento inicial fue remitido a Fisioterapeuta o Terapeuta Ocupacional no especializado o a Terapeuta de Mano y Miembro Superior, con el fin de conocer la pertinencia en la remisión para su intervención.

Como resultado de los diferentes procesos de transformación de las poblaciones en aspectos sociales, culturales, económicos y demográficos, las condiciones de salud son cada vez más complejas y de la misma forma los requerimientos de atención de ésta son mayores (15).

En el entorno colombiano la violencia interna hace que muchas lesiones sean de gran complejidad y su atención sobrepase la perspec- tiva de intervención básica, por lo cual se hace necesario direccionar los esfuerzos al cubrimiento de las deficiencias en salud en personas con traumas osteomusculares y neurológicos, que de no tratarse adecuadamente podrían comprometer seriamente su función y que en sociedades diferentes, habrían sido prevenibles $(16,17)$.

De acuerdo con el Instituto Nacional de Medicina Legal (18), los años de vida saludable perdidos (AVISA) En 2009, fueron 184.217, que corresponde a 1.3 años para cada individuo lesionado y aunque el informe no especifica las lesiones de miembro superior, éstas hacen parte de las estadísticas nacionales.

El miembro superior del ser humano juega un papel muy importante en la función de las personas, por lo cual las patologías presentes comprometen no sólo las estructuras anatómicas y su fisiología, sino que pueden llevar a limitación funcional, a restricción en su participación social o en último caso a discapacidad, determinadas por lo establecido en la Clasificación de Funcionamiento y Discapacidad (CIF) como la sumatoria del compromiso estructural, del funcionamiento y del rol social de cada individuo con factores ambientales y personales propios $(19,20)$.

En traumas moderados o severos, patologías asociadas a la actividad laboral o alteraciones sistémicas que comprometen el miembro superior, se requiere de terapeutas especializados (pertinencia) para mejorar o recuperar la función del usuario abordado además de la oportunidad en la intervención.

Está demostrado que la iatrogenia en su intervención lleva a compromisos serios en la funcionalidad de quien la recibe $(21,22)$. Para la recuperación de éstas lesiones es necesaria la pertinente formación académica del profesional, y la suficiente experticia para realizar una acertada intervención médica y terapéutica (23), herramientas que sólo se obtienen cuando se ha trabajado en equipos interdisciplinarios especializados en la temática específica o cuando la academia certifica la formación posgradual que va a permitir la interacción en la rehabilitación de quien lo requiera.

En Colombia los profesionales que históricamente han abordado la terapéutica para rehabilitar los usuarios con patología osteomuscular y específicamente de miembro superior son los Fisioterapeutas y los Terapeutas Ocupacionales, y aunque hay varios de éstos profesionales destacados por su estudio y dedicación en el área, sólo hasta la fecha, aproximadamente 100 de éstos están certificados como Especialistas en Rehabilitación de la Mano y Miembro Superior, por la Escuela Colombiana de Rehabilitación (ECR), de los aproximadamente 16.00o Fisioterapeutas y 1.200 Terapeutas Ocupacionales (24), egresados de los diferentes programas de pregrado a nivel nacional. También se reconocen como especialistas en terapia de mano, los Fisioterapeutas y Terapeutas Ocupacionales que tienen reconocida experticia (mayor de tres años) por su dedicación específica y profundización en el conocimiento de ésta área, o los que son certificados como Terapeutas de Mano, por instituciones educativas extranjeras.

Por lo anteriormente abordado se hizo la presente investigación en Colombia, para establecer la oportunidad que tienen los pacientes con patología de miembro superior para acceder al tratamiento 
terapéutico en un tiempo determinado y a la terapéutica especializada cuando así lo demanden y la pertinencia en éste contexto aplica para la atención sin restricción cuando lo requiera el estado de salud de un individuo, por parte de un profesional especializado y en éste caso de un terapeuta para minimizar los riesgos de error en la intervención de los pacientes.

\section{METODOLOGÍA}

El presente estudio es exploratorio-descriptivo (25) en el que se tomaron datos en instituciones de salud de 60 pacientes afiliados al régimen subsidiado, 135 de régimen contributivo y 82 con medicina prepagada, que consultaron en varias ciudades de Colombia entre los meses de abril y julio de 2011, por patología de miembro superior que comprometió su función, sin restricción de edad, sexo ni estrato socio económico. Se excluyeron del estudio los pacientes con patología de miembro superior que no comprometía su función, los que fueron atendidos en tiempos diferentes a los establecidos en el estudio y los pacientes que pertenecieran a regímenes especiales de salud (Fuerzas Militares y Policía Nacional).

Para la toma de los datos se elaboró un formato con 13 ítems, que permitió establecer la pertinencia y la oportunidad en el tratamiento terapéutico recibido.

La pertinencia se evaluó con los reactivos 8 y 9 del formato de recolección de datos

La oportunidad se midió con los reactivos 10, 11 y 12 del formato de recolección de datos.

Dicho formato fue sometido a pilotaje por la autora del proyecto y por los 15 estudiantes de la Especialización de Rehabilitación de la Mano y el Miembro Superior de la ECR, VI Cohorte, quienes tomaron los datos en instituciones de salud de sus ciudades de origen que fueron Bogotá, Cali, Palmira, Bucaramanga, Cúcuta, Villavicencio, Medellín, Sandoná y San Pablo (Nariño).

Luego de los ajustes realizados al formato los asistentes iniciaron el proceso para recolección de la muestra e hicieron contactos con las instituciones de salud que aceptaron hacer parte del estudio, para que les autorizaran la toma de datos de los pacientes de los diferentes regímenes de salud, incluidos en la investigación.

Posterior a su aprobación y antes de iniciar la toma de la muestra se firmó el acuerdo de confidencialidad entre cada institución de salud y la ECR, el compromiso de confidencialidad por parte del asistente de investigación y se envió el formato de consentimiento informado para ser firmado por cada paciente contactado y que aceptó participar en la investigación. Lo anterior se hizo como consideración ética para recolección de la información, tanto la suministrada por la Institución (estadísticas, RIPS), como la de los pacientes (Historia Clínica y contacto personal).

Seguido de lo anterior se accedió a las estadísticas de los meses entre abril y julio de 2011 y de allí se tomaron todos los pacientes nuevos que fueron atendidos con patología de miembro superior y de éste grupo, se seleccionaron según el régimen de salud por el que ingresaron (subsidiado, contributivo o medicina prepagada), y con base en el criterio y experticia de los candidatos a especialistas en Rehabilitación de la Mano y el Miembro Superior de la ECR, se seleccionaron los que presentaron patología de miembro superior que comprometía su función.

Posteriormente se contactó telefónicamente a los usuarios seleccionados con el fin de pedirles su aceptación para participar en la investigación y acordar la firma del consentimiento informado. A partir de ese proceso se registraron los datos en el formato elaborado para tal fin y luego se ingresó la información en una base de datos en Excel para su posterior análisis.

Instrumento de recolección de datos

El formato para recolección de los datos incluyó los registros de historia clínica, los personales de cada paciente y los antecedentes de la patología, en los reactivos del 1 al 10.

Para evaluar oportunidad se hicieron tres preguntas, dos para pertinencia y una complementaria de la información para conocer la satisfacción del paciente.

El total de preguntas fue de 17 luego del pilotaje al que fue sometido el instrumento.

\section{RESULTADOS}

Luego de hacer el análisis de los datos se encontró que el promedio de género en la muestra seleccionada, fue similar en los tres regímenes, con un de $58 \%$ de mujeres y $42 \%$ de hombres.

El mayor porcentaje de edad de los pacientes en los tres regímenes, fue en mayores de 56 años e inferior en menores de 25 años.

Se estableció que la mayoría de los pacientes que consultaron en los regímenes de salud subsidiado y contributivo, inicialmente fueron atendidos por médico general mientras que el $50 \%$ de los afiliados a medicina prepagada, tuvo la atención inicial por un especialista en Ortopedia y el $21 \%$ por especialista de Mano y Miembro Superior, esto es que el $71 \%$ de éstos últimos pacientes recibió intervención inicial pertinente para su lesión. (Tabla 1).

\begin{tabular}{|c|c|c|c|}
\hline CONSULTA & SUBSIDIADO & CONTRIBUTIVO & PREPAGADA \\
\hline General & $80 \%$ & $66 \%$ & $21 \%$ \\
\hline Ortopedia & $15 \%$ & $17 \%$ & $50 \%$ \\
\hline $\begin{array}{c}\text { Mano y Ms } \\
\text { Cirujano } \\
\text { Plástico }\end{array}$ & $2 \%$ & $1 \%$ & $21 \%$ \\
\hline Otro & $0 \%$ & $1 \%$ & $0 \%$ \\
\hline
\end{tabular}

Tabla 1. Atención médica inicial según regimen de salud. 
Posterior a la consulta inicial, en los tres regímenes, el paciente fue remitido a un especialista para el manejo de su lesión en porcentajes superiores al $60 \%$. El 21\% de los afiliados a medicina prepagada fueron remitidos a medicina especializada en Mano y Miembro Superior, mientras que ninguno de los subsidiados la recibió. (Tabla 2).

\begin{tabular}{|l|c|c|c|}
\hline \multicolumn{1}{c|}{$\begin{array}{c}\text { TIPO DE } \\
\text { ESPECIALISTA }\end{array}$} & SUBSIDIADO & CONTRIBUTIVO & PREPAGADA \\
\hline Fisiatra & $11 \%$ & $21 \%$ & $16 \%$ \\
\hline Ortopedista & $76 \%$ & $62 \%$ & $63 \%$ \\
\hline $\begin{array}{l}\text { Cirujano } \\
\text { Plástico }\end{array}$ & $5 \%$ & $9 \%$ & $0 \%$ \\
\hline Neurólogo & $8 \%$ & $1 \%$ & $0 \%$ \\
\hline $\begin{array}{l}\text { Especialista } \\
\text { En MMSS }\end{array}$ & $0 \%$ & $3 \%$ & $21 \%$ \\
\hline NR & $0 \%$ & $4 \%$ & $0 \%$ \\
\hline
\end{tabular}

Tabla 2. Remisión a Especialista según régimen de salud

A la consulta de si fue remitido a Fisioterapia no especializada, el $98 \%$ de los pacientes subsidiados respondió que si, al igual que el $100 \%$ en los otros dos regímenes.

Los datos reportaron que ninguno de los pacientes de la muestra tuvo intervención terapéutica especializada.

La oportunidad se evaluó como muy oportuna cuando se dio la remisión a terapéutica entre el $1^{\circ}$ y el $5^{\circ}$ día posterior a la lesión, oportuna cuando se dio entre el $6^{\circ}$ y el $10^{\circ}$ día, poco oportuna entre 11 y 15 días y no oportuna, después de 15 días. También se incluyó como oportunidad el número de terapias asignadas para tratamiento.

Los resultados en esta variable reportaron que al $68 \%$ de los pacientes del régimen contributivo y al $94 \%$ de los afiliados a medicina prepagada se les asignó la cita a terapéutica no especializada en los primeros cinco días, y en el mismo tiempo al $52 \%$ de los pacientes subsidiados. El $42 \%$ de los pacientes del régimen subsidiado fueron remitidos a terapia entre los 6 y 10 días posteriores a la consulta inicial. (Tabla 3 )

TIEMPO DE ATENCIÓN ENTRE CONSULTA Y ASIGNACIÓN

\begin{tabular}{|c|c|c|c|}
\hline DIAS & SUBSIDIADO & CONTIBUTIVO & PREPAGADA \\
\hline 1 a 5 & $52 \%$ & $68 \%$ & $94 \%$ \\
\hline 6 a 10 & $42 \%$ & $19 \%$ & $6 \%$ \\
\hline 11 a 15 & $6 \%$ & $9 \%$ & $0 \%$ \\
\hline$>15$ & $0 \%$ & $4 \%$ & $0 \%$ \\
\hline
\end{tabular}

Tabla 3. Relación entre la consulta inicial y la asignación de la cita según régimen de salud
Al $58 \%$ de los pacientes subsidiados, al $42 \%$ de los contributivos y al $84 \%$ de medicina prepagada les fueron asignadas entre 6 y 10 terapias para tratamiento. Con igualdad del $20 \%$ a los regímenes subsidiado y contributivo le fueron autorizadas entre 11 y 15 terapias; al $24 \%$ del régimen contributivo le autorizaron más de 15 sesiones de tratamiento.

En el régimen contributivo un $56 \%$ de los pacientes y en la medicina prepagada un $46 \%$, consultaron por presentar patología de miembro superior por trauma acumulativo, mientras que en el régimen subsidiado el $44 \%$ lo hizo por trauma directo. (Tabla 4)

\begin{tabular}{|c|c|c|c|}
\hline \multicolumn{4}{|c|}{ TERAPIAS AUTORIZADAS } \\
\hline $\begin{array}{c}\text { NÚMERO DE } \\
\text { TERAPIAS }\end{array}$ & SUBSIDIADO & CONTIBUTIVO & PREPAGADA \\
\hline 1 a 5 & $3 \%$ & $13 \%$ & $1 \%$ \\
\hline 6 a 10 & $58 \%$ & $42 \%$ & $84 \%$ \\
\hline 11 a 15 & $20 \%$ & $20 \%$ & $7 \%$ \\
\hline$>15$ & $18 \%$ & $24 \%$ & $7 \%$ \\
\hline
\end{tabular}

Tabla 4. Cantidad de terapias autorizadas inicialmente según régimen de salud

\section{DISCUSIÓN}

En cuanto a pertinencia se destaca que los regímenes subsidiado y contributivo tienen menos acceso a un médico especializado, que los afiliados a medicina prepagada quienes sí la reciben en la mitad de los casos.

Cabe resaltar que en ninguno de los regímenes de la muestra se dio remisión a terapéutica especializada, lo cual debe ser analizado en nuevas investigaciones que establezcan la calidad de los tratamientos llevados a cabo y también determinen si no hay recurso humano suficiente que supla las necesidades de atención de pacientes con patología de miembro superior.

Respecto de la oportunidad es destacable que en el $52 \%$ de los casos del régimen subsidiado, en el $68 \%$ de los contributivos y en el $94 \%$ de los afiliados a medicina prepagada, fue muy oportuna la remisión a terapéutica no especializada y en el $42 \%$ de los afiliados a medicina prepagada fue oportuna.

Autores como Gloria Molina (26) han concluido que a pesar de que el nuevo modelo de aseguramiento en salud en Colombia ha dado oportunidad a las personas de bajo recurso económico de acceder a los diferentes servicios de salud, son muchas las voces que hablan de la falta de oportunidad y eficiencia en la prestación de los servicios de salud.

Es también cuestionable que aunque hay incremento en cobertura en afiliación hay inequidad en la prestación de los servicios, pues priman los intereses económicos de las empresas prestadoras de salud sobre la obligación que tienen asignada por el SGSS, de promover, recuperar y mantener la salud de sus afiliados (26). 
La nueva ética en la práctica de salud y del SGSS, debe afianzarse en una concepción de justicia por el respeto y la libertad del paciente, en la igualdad de oportunidades y acceso a los servicios de salud, según lo necesite (27), pues la idoneidad en su intervención, minimiza los riesgos de iatrogenia, en beneficio de su salud.

Los gremios en salud deben unirse para instar al Gobierno Nacional a que en la atención de las personas con compromiso de su salud no tengan injerencia los aspectos económicos, sino que primen los principios del derecho a la salud consagrados en la Constitución Nacional de Colombia.

\section{CONCLUSIONES}

Es oportuna la remisión a terapéutica no especializada mas no es pertinente la atención terapéutica puesto que el total de los pacien- tes fue atendido por terapeutas no especializados a pesar de tener patología de miembro superior que comprometía su función.

Es importante realizar nuevos estudios que analicen las intervenciones terapéuticas dadas a los pacientes con compromiso de su función por lesión de miembro superior para concluir si son idóneos o si es necesario promover la formación académica de los profesionales con posgrados que fundamenten su acción para la completa reintegración de los pacientes a su entorno social.

Fue limitante en la presente investigación la imposibilidad de desplazamiento de la investigadora principal a las diferentes ciudades en donde se recolectó la información. 


\section{Referencia: muy importante en la literatura científica;
estas permiten conocermás sobre los autores y
mantener vivas sus voces dentro del texto.}

1 Ministerio de Salud y Protección Social. República de Colombia. Ley 100. 1993.

2 Darwin. Ley de correlación del crecimiento. Citado por Monreal G. Ricardo. La mano, origen, evolución y su papel en la sociedad. Rev. Cubana Ortopedia y Traumatología Vol.21 No.2. La Habana Cuba. 2007

3 Sen Amartya. ¿Por qué la equidad en salud?. Revista Panamericana de Salud Pública. Vol. 11 No. 5-6 2002. Washington

4 Centro de Prensa de la Organización Mundial de la Salud. 63 ${ }^{\mathrm{a}}$ Asamblea Mundial de La Salud. Ginebra, Suiza. 17-21 de mayo de 2010.

5 Programa de las Naciones Unidas para el Desarrollo en Colombia (PNUD). Informe nacional de Desarrollo Humano 2003: Callejón con salida.

6 Organización Mundial de la Salud. Carta de Ottawa para la promoción de la salud. 1986

7 Moran M. Governing the health care state: a comparative study of the United. Kingdom, The United States and Germany. Manchester and New York NY, Manchester Universitu Press, 1999

8 Congreso de La República. Ley 1122. Modificaciones en el Sistema General de Seguridad Social en Salud. Bogotá enero 2007.

9 Congreso de La República. Decreto 1011. Ministerio de Protección Social. Colombia. Sistema Obligatorio de Garantía de Calidad (SOGCS) de la Atención de Salud del Sistema General de Seguridad Social en Salud. Abril 3 de 2006

10 Zambrano A., Ramírez M., "¿Qué muestran las encuestas de calidad de vida sobre el sistema de salud en Colombia?" Revista Salud Pública Vol. 24 No.1 Rio de Janeiro. Enero 2008.

11 Vargas J, Molina G., Acceso a los servicios de salud en seis ciudades de Colombia: limitaciones y consecuencias. Rev. Fac. Nac. Salud PúbliCa. 2009:27(2) 121-130.

12 Gazzetta Offíciale della Repubblica Italiana "Carta de los servicios públicos sanitarios" en, Suplemento ordinario n ${ }^{\circ} 125,31$ de mayo de 1995.

13 Mejía Villegas Darío y equipo investigativo del Programa de Salud y Seguridad Social de la Defensoría del Pueblo. "Evaluación de los servicios de Salud que brindan las Empresas Promotoras de Salud". Colombia. 2009.

14 Caja Nacional de Previsión (Cajanal). Resolución nº 000707. Atención en servicios de salud. Servicio tiempo para la atención. Bogotá. Abril 3 de 1996.
15 Mejía A., Sánchez A., Tamayo Juan, Calidad y visibilidad. Rev. Salud Pública. Vol ९ No.1. Bogotá 2007

16 Vélez Rojas Hernando. "Violencia y cirugía en Colombia. Conferencia en XXIX Congreso Nacional "Avances en Cirugía" Santa Marta. Colombia. Agosto 2003

17 DANE. Seguridad Social en Salud y Pensiones. Enero-Marzo 2010. Bogotá

18 Vargas Roa Ana M., Algunas reflexiones sobre la relación entre capital social y violencia interpersonal. Publicación del Instituto de Medicina Legal y Ciencias Forenses. Bogotá. 2010. Pag.80

19 Funcionamiento y Discapacidad: CIF. Revista Española de Salud Pública. 2009; Vol. 83.

20 Rodríguez Lozano Rafael. Fisioterapia y discapacidad: hacia un modelo de atención integral. Revista de Fisioterapia. 2008;30:163. España

21 Hunter James, Rehabilitation of the hand and upper extremity. Six edition. Ed. Mosby. 2011. Philadelphia.

22 Gómez Aceituno, Verresen Ximénez, Intervención terapéutica interdisciplinaria en la mano artrósica. A propósito de dos casos. Revista Iberoamericana de Fisioterapia y Kinesiologia. Vol. 10:103-11 No.o2. Julio 2007

23 Yancosek, Kathleen E. "Management of dominante upper extremity injuries: a survey of practice patterns". Journal of hand therapy. Octubre de 2011.

24 Observatorio Laboral. (Sitio de internet) disponible en: www.graduadoscolombia.edu.co. Acceso el 13 de mayo de 2011.

25 Hernández Sampieri R., Metodología de la investigación. $4^{a}$. Edición. Ed. Mc Graw Hill. Méjico. 2006.

26 Molina M., Gloria, Muñoz E., Iván Felipe, Ramírez G. Andrés, Dilemas en las decisiones en la atención en Salud. Grupo de investigación Facultad Nacional de Salud Pública. Universidad de Antioquia. Ed. Instituto de Estudios del Ministerio Público. Bogotá Colombia. Enero 2009.

27 Vega Romero, Román. Dilemas éticos contemporáneos en salud el caso colombiano desde la perspectiva de la justicia social. Revista Gerencia y Políticas de Salud [en línea] 2002, 1 (septiembre): [fecha de consulta: 8 de diciembre de 2011]Disponibleen: <http://redalyc.uaemex.mx/redalyc/src/inicio/ArtPdfRed.jsp?iCve=54510206> ISSN $1657-7027$ 